\title{
RG study of a non-local sine-Gordon model
}

\author{
Carlos M. Naón ${ }^{\mathrm{a}, \mathrm{b}}$, Mariano J. Salvay ${ }^{\mathrm{a}, \mathrm{b}}$ \\ ${ }^{a}$ Instituto de Física La Plata, Departamento de Física, Facultad de Ciencias Exactas, \\ Universidad Nacional de La Plata, CC 67, 1900 La Plata, Argentina \\ ${ }^{\mathrm{b}}$ Consejo Nacional de Investigaciones Cientificas y Técnicas, Argentina
}

Received 3 December 2002; received in revised form 21 March 2003; accepted 25 April 2003

\begin{abstract}
We study a non-local version of the sine-Gordon model connected to a many-body system with backward and umklapp scattering processes. Using renormalization group methods we derive the flow equations for the couplings and show how non-locality affects the gap in the spectrum of charge-density excitations. We compare our results with previous predictions obtained through the self-consistent harmonic approximation.
\end{abstract}

(c) 2003 Elsevier B.V. All rights reserved.

PACS: 11.10.Lm; 05.30.Fk

Keywords: Field theory; Non-local; Renormalization group

\section{Introduction}

In general, Quantum Field Theories have been built in the context of local models. However, there exist physical situations that lead to non-local interactions in a straightforward way. Let us mention, for instance, Wheeler and Feynman's description of charged particles [1], string theories with non-local vertices [2], and non-local kinetic terms that appear when bosonizing fermions in $2+1$ dimensions $[3,4]$. As we shall see, several recently considered non-local field theories are related to the study of electronic systems in one spatial dimension (1D) [5,6]. Indeed, in recent years the physics of 1D systems of strongly correlated particles has become a very interesting subject since one can take advantage of the simplicity of the models at hand and, at the same time, expect to make contact with experiments. For instance, the recently discovered carbon nanotubes are perfect experimental

E-mail addresses: naon@fisica.unlp.edu.ar (C.M. Naón), salvay@fisica.unlp.edu.ar (M.J. Salvay). 
realizations of $1 \mathrm{D}$ conductors [7]. On the other hand, as the dimensionality of a system decreases, charge screening effects become less important and the long-range interaction between electrons is expected to play a central role in determining the properties of the system. In fact, from a theoretical point of view the effects of long-range interactions have been recently discussed in connection to several problems such as the Fermi-edge singularity [8], the insulator-metal transition [9], the role of the lattice through umklapp scattering and size dependent effects [10], etc. In the specific context of carbon nanotubes, a lowenergy theory including Coulomb interactions has been also recently derived and analyzed [11]. Non-local fermionic models have been also used in the study of fluctuation effects in low-dimensional Spin-Peierls systems [12].

As shown in [13], starting from a non-local and non-covariant version of the Thirring model [14], in which the fermionic densities and currents are coupled through bilocal, distance-dependent potentials, one can make direct contact with the " $g$-ology" model [15] currently used to describe different scattering processes characterized by coupling functions $g_{1}, g_{2}, g_{3}$ and $g_{4}$. When one bosonizes this theory by either operational or functional methods, due to the contributions of $g_{1}$ (backscattering) [16] and $g_{3}$ (umklapp) [17] one finds an even more drastic departure from the local case. Indeed, instead of the well-known integrable sine-Gordon model (SG) one gets a non-local extension of it, which, as far as we know, is not exactly solvable. Recently, in Ref. [13], the physical content of this model was explored by using the self-consistent harmonic approximation (SCHA) [18]. As it is well known the SCHA is a non-controlled approximation, i.e., there is no perturbative parameter involved. It is then desirable to have an alternative analysis of this problem. This is the main motivation of the present work. We will apply the renormalization group (RG) technique [19], usually employed in local cases, to the non-local sine-Gordon model (NLSG) mentioned above. For simplicity we shall assume that non-locality plays a role only in umklapp interactions $\left(g_{3}\right)$ whereas all other potentials are local, i.e., proportional to delta functions. In Section 2 we briefly show how the NLSG action is obtained from the non-local Thirring model. In Section 3 we derive the RG equations and compute the gap in the charge-density spectrum. This allows us to determine the effect of non-contact $g_{3}$ couplings. Finally, in Section 4, we discuss our results.

\section{The model}

Let us sketch the derivation of the NLSG action. We start from the fermionic $(1+1)$ dimensional Quantum Field Theory with Euclidean action given by

$$
S=S_{0}+S_{f s}+S_{b s}+S_{u s}
$$

where

$$
S_{0}=\int d^{2} x \bar{\Psi} i \not \partial \Psi
$$

is the unperturbed action associated to a linearized free dispersion relation. The contributions of the different scattering processes can be written as

$$
S_{f s}=-\frac{g^{2}}{2} \int d^{2} x d^{2} y\left(\bar{\Psi}_{\gamma_{\mu}} \Psi\right)(x) V_{(\mu)}(x, y)\left(\bar{\Psi} \gamma_{\mu} \Psi\right)(y)
$$


and

$$
S_{b s}+S_{u s}=-\frac{g^{\prime 2}}{2} \int d^{2} x d^{2} y\left(\bar{\Psi} \Gamma_{\mu} \Psi\right)(x) U_{(\mu)}(x, y)\left(\bar{\Psi} \Gamma_{\mu} \Psi\right)(y),
$$

where the $\gamma_{\mu}^{\prime} s$ are the usual two-dimensional Dirac matrices and $\Gamma_{0}=1, \Gamma_{1}=\gamma_{5}$. The coupling potentials $V_{(\mu)}$ and $U_{(\mu)}$ are assumed to depend on the distance $|x-y|$ and can be expressed in terms of " $g$-ology" parameters as

$$
\begin{aligned}
& V_{(0)}(x, y)=\frac{1}{g^{2}}\left(g_{2}+g_{4}\right)(x, y), \\
& V_{(1)}(x, y)=\frac{1}{g^{2}}\left(g_{2}-g_{4}\right)(x, y), \\
& U_{(0)}(x, y)=\frac{1}{g^{\prime 2}}\left(g_{3}+g_{1}\right)(x, y), \\
& U_{(1)}(x, y)=\frac{1}{g^{\prime 2}}\left(g_{3}-g_{1}\right)(x, y) .
\end{aligned}
$$

In the above equations $g$ and $g^{\prime}$ are just numerical constants that could be set equal to one. We keep them to facilitate comparison of our results with those corresponding to the usual Thirring model. Indeed, this case is obtained by choosing $g^{\prime}=0$ and $V_{(0)}(x, y)=$ $V_{(1)}(x, y)=\delta^{2}(x-y)$. On the other hand, the non-covariant limit $g^{\prime}=0, V_{(1)}(x, y)=0$ gives one version $\left(g_{2}=g_{4}\right)$ of the TL model [20].

The terms in the action containing $g_{2}$ and $g_{4}$ represent forward scattering events, in which the associated momentum transfer is small. In the $g_{2}$ processes the two branches (left- and right-moving particles) are coupled, whereas in the $g_{4}$ processes all four participating electrons belong to the same branch. On the other hand, $g_{1}$ and $g_{3}$ are related to scattering diagrams with larger momentum transfers of the order of $2 k_{F}$ (bs) and $4 k_{F}$ (us) respectively (this last contribution is important only if the band is half-filled). For simplicity, throughout this paper we will consider spinless electrons. The extension of our results to the spin-1/2 case with spin-flipping interactions, though not trivial, could be done by following the lines of Ref. [21].

At this point we consider the partition function $\mathcal{Z}$ expressed as a functional integral over fermionic variables. The implementation of a generalized Hubbard-Stratonovich identity [6] allows to write $\mathcal{Z}$ in terms of a fermionic determinant. Although this determinant is highly non-trivial, one can combine a chiral change in the fermionic path-integral measure with a formal expansion in $g^{\prime}$ in order to obtain a bosonic representation (see Ref. [13] for details). One thus establishes an equivalence between the original fermionic action and the following bosonic action depending on five scalars $\Phi, \eta, C_{0}, C_{1}$ and $\varphi$ :

$$
\begin{aligned}
S_{\mathrm{bos}}=\int \frac{d^{2} p}{(2 \pi)^{2}}[\Phi(p) \Phi(-p) A(p)+\eta(p) \eta(-p) B(p)+\Phi(p) \eta(-p) C(p) \\
\left.+\varphi(p) \varphi(-p) \frac{p^{2}}{2}\right] \\
+\frac{1}{2} \int d^{2} x d^{2} y C_{\mu}(x) U_{(\mu)}^{-1}(x, y) C_{\mu}(y)
\end{aligned}
$$




$$
+\frac{g^{\prime} \Lambda c}{\pi} \int d^{2} x\left[C_{(0)}(x) f_{0}(x)+i C_{(1)}(x) f_{1}(x)\right]
$$

where

$$
\begin{aligned}
& A(p)=\frac{1}{2}\left[p_{0}^{2} \widehat{V}_{(1)}^{-1}(p)+p_{1}^{2}\left(\widehat{V}_{(0)}^{-1}(p)+\frac{g^{2}}{\pi}\right)\right], \\
& B(p)=\frac{1}{2}\left[p_{0}^{2} \widehat{V}_{(0)}^{-1}(p)+p_{1}^{2}\left(\widehat{V}_{(1)}^{-1}(p)-\frac{g^{2}}{\pi}\right)\right], \\
& C(p)=p_{0} p_{1}\left(\widehat{V}_{(0)}^{-1}(p)-\widehat{V}_{(1)}^{-1}(p)+\frac{g^{2}}{\pi}\right),
\end{aligned}
$$

and

$$
\begin{aligned}
& f_{0}(x)=\cos ((\sqrt{4 \pi} \varphi-2 i g \Phi)(x)), \\
& f_{1}(x)=\sin ((\sqrt{4 \pi} \varphi-2 i g \Phi)(x)) .
\end{aligned}
$$

Since the integrals in $C_{0}$ and $C_{1}$ are quadratic these fields are easily integrated out and one gets

$$
\mathcal{Z}=\mathcal{N} \int \mathcal{D} \Phi \mathcal{D} \eta \mathcal{D} \varphi e^{-S_{\text {eff }}[\Phi, \eta, \varphi]}
$$

with

$$
S_{\mathrm{eff}}[\Phi, \eta, \varphi]=S_{0}+S_{\mathrm{int}},
$$

where

$$
\begin{aligned}
& S_{0}=\int \frac{d^{2} p}{(2 \pi)^{2}}[\Phi(p) \Phi(-p) A(p)+\eta(p) \eta(-p) B(p)+\Phi(p) \eta(-p) C(p) \\
&\left.+\varphi(p) \varphi(-p) \frac{p^{2}}{2}\right], \\
& S_{\mathrm{int}}=- \frac{(\Lambda c)^{2}}{2 \pi^{2}} \int d^{2} x d^{2} y g_{1}(x, y) \cos [\sqrt{4 \pi}(\varphi(x)-\varphi(y))-2 i g(\Phi(x)-\Phi(y))] \\
&-\frac{(\Lambda c)^{2}}{2 \pi^{2}} \int d^{2} x d^{2} y g_{3}(x, y) \cos [\sqrt{4 \pi}(\varphi(x)+\varphi(y))-2 i g(\Phi(x)+\Phi(y))] .
\end{aligned}
$$

It is now convenient to diagonalize the quadratic part of the effective action by introducing the fields $\zeta, \chi$ and $\phi$ :

$$
\begin{aligned}
& \Phi=\frac{i \zeta}{\tilde{g}}+\frac{2 i \tilde{g} B p^{2}}{\Delta+2 B \tilde{g}^{2} p^{2}} \phi, \\
& \eta=\frac{-i C}{2 B \tilde{g}} \zeta-\frac{i \tilde{g} C p^{2}}{\Delta+2 B \tilde{g}^{2} p^{2}} \phi+\frac{1}{\tilde{g}} \chi, \\
& \varphi=-\zeta+\frac{\Delta}{\Delta+2 B \tilde{g}^{2} p^{2}} \phi,
\end{aligned}
$$


where we have defined $\tilde{g}^{2}=g^{2} / \pi$ and $\Delta(p)=C(p)^{2}-4 A(p) B(p)$. We then obtain

$$
\begin{aligned}
S_{0}=\frac{1}{2} \int \frac{d^{2} p}{(2 \pi)^{2}}\left[\zeta(p)\left(p^{2}+\frac{\Delta}{2 B \tilde{g}^{2}}\right) \zeta(-p)+\chi(p) \frac{2 B}{\tilde{g}^{2}} \chi(-p)\right. \\
\left.+\phi(p) \frac{p^{2} \Delta}{\Delta+2 B \tilde{g}^{2} p^{2}} \phi(-p)\right], \\
S_{\mathrm{int}}=-\frac{(\Lambda c)^{2}}{2 \pi^{2}} \int d^{2} x d^{2} y g_{1}(x, y) \cos \sqrt{4 \pi}[\phi(x)-\phi(y)] \\
-\frac{(\Lambda c)^{2}}{2 \pi^{2}} \int d^{2} x d^{2} y g_{3}(x, y) \cos \sqrt{4 \pi}[\phi(x)+\phi(y)] .
\end{aligned}
$$

One can see that the $\zeta$ and $\chi$ fields become completely decoupled from $\phi$. Moreover, it becomes apparent that the $\phi$-dependent piece of the action $S_{\text {int }}$ is the only one containing potentially relevant contributions (i.e., gapped modes).

\section{RG treatment of the non-local umklapp coupling}

In this section we shall focus our attention on the non-local action derived above. For simplicity, from now on we will consider the case in which $g_{2}(p)$ and $g_{4}(p)$ are constants (local forward scattering) and $g_{1}=0, g_{3} \neq 0$, i.e., a pure non-local umklapp interaction. Thus, we start from the action

$$
\begin{aligned}
S[\phi]= & \int \frac{d^{2} p}{(2 \pi)^{2}} \phi(p) \frac{F(p)}{2} \phi(-p) \\
& -\frac{(\Lambda c)^{2}}{2 \pi^{2}} \int d^{2} x d^{2} y g_{3}(x, y) \cos \sqrt{4 \pi}[\phi(x)+\phi(y)]
\end{aligned}
$$

with

$$
\begin{aligned}
& F(p)=\frac{1}{K v}\left(p_{0}^{2}+v^{2} p_{1}^{2}\right), \\
& K=\sqrt{\frac{1+g_{4} / \pi-g_{2} / \pi}{1+g_{4} / \pi+g_{2} / \pi},} \\
& v=\sqrt{\left(1+\frac{g_{4}}{\pi}+\frac{g_{2}}{\pi}\right)\left(1+\frac{g_{4}}{\pi}-\frac{g_{2}}{\pi}\right)},
\end{aligned}
$$

where we have now expressed all formulae in terms of $g$ coupling functions. In the local case $\left(g_{3}=\delta^{2}(x-y)\right)$ the action (3.1) corresponds to the well-known sine-Gordon model, which is an integrable, exactly solvable field theory. In particular, a RG analysis shows that the "stiffness constant", $K$ has to be lower than 0.5 in order to have a relevant cosine interaction, i.e., to have a gap in the spectrum. Recently, by reinterpreting Bethe ansatz results, Zamolodchikov obtained the exact expression for this gap [22]. Unfortunately, as far as we know, the present non local version of the theory is not exactly soluble and one 
is then forced to consider an approximation. In Ref. [13] a SCHA was employed in order to estimate the energy gap. But this is a non-controlled, non-perturbative approximation. Besides this general disadvantage, the implementation of the SCHA technique led to a set of coupled algebraic equations that could be numerically solved only for very weak non-locality. It is then natural to try another method to attack the problem and eventually improve the approximation. Let us consider the Wilsonian approach to the RG (see, for instance, [19]). First of all we restrict our analysis to a non-local interaction of the form:

$$
g_{3}(x-y)=g_{3}\left(x_{1}-y_{1}\right) \delta\left(x_{0}-y_{0}\right),
$$

with

$$
g_{3}\left(x_{1}\right)=\lambda_{0} \delta\left(x_{1}\right)-\frac{\epsilon_{0}}{\Lambda^{2}} \partial_{1}^{2} \delta\left(x_{1}\right),
$$

where $\partial_{1}^{2} \delta^{2}(x)$ is the second derivative of the delta function with respect to $x_{1}$. At this point it is worth mentioning that $\mathrm{RG}$ calculations involving fermionic non-local interactions already exist in the literature. For instance, the authors of Ref. [12] used a Jordan-Wigner fermion representation for the $1 \mathrm{D}$ Heisenberg-Ising model which includes not only a non-local fermion-fermion interaction but also a linear fermion-lattice coupling. Due to the curvature of the band, the non-trivial fermion-lattice coupling and the presence of both forward and umklapp scattering, comparison of their RG equations with ours is not straightforward. Let us point out, however, that in [12] fermions corresponding to nearestneighbor lattice sites interact through a potential of the form $g(q)=C_{1} \times \cos q$, i.e., a unique constant $C_{1}$ is associated to $g(q)$. As shown in the above equation (3.6), in the present work we are interested in a coupling which depends at least on two constants $\lambda$ and $\epsilon$. In fact, the derivation of RG equations for these coupling constants will be our next task.

In condensed matter problems one is usually interested in the physics at long distances, compared to a lattice spacing of the order of $\Lambda^{-1}$. Since, in momentum space, this corresponds to small $k_{1}=\mathbf{k}$, it is natural to consider correlations between fields with momenta $0<k<\Lambda / s$, with $s$ very large. These are the so-called "slow modes" $\phi_{<}$. On the other hand, the "fast modes" $\phi_{>}$are those carrying momenta that satisfy $\Lambda / s \leqslant k \leqslant \Lambda$. In the present approach to RG these fast modes are integrated in the path integral framework, giving rise to an effective theory depending only on slow modes. From this action, in turn, one can read the flow equations for the couplings. Indeed, writing the initial action as $S_{0}-S_{\text {int }}$, to first order, after a suitable rescaling of coordinates and momenta and a redefinition of the fields (see Appendix A for details) we obtain the following relationship between the original and RG transformed actions:

$$
\begin{aligned}
S_{\text {int }}= & \frac{(\Lambda c)^{2}}{2 \pi^{2}} \int d^{2} x d^{2} y\left(\left[\lambda_{0}+\lambda_{0}(2-4 K) t-2 K \epsilon_{0} t\right] \delta^{2}(x-y)\right. \\
& \left.\quad-\frac{\epsilon-4 K \epsilon t}{\Lambda^{2}} \partial_{1}^{2} \delta^{2}(x-y)\right) \cos \sqrt{4 \pi}(\phi(x)+\phi(y)) \\
= & \frac{(\Lambda c)^{2}}{2 \pi^{2}} \int d^{2} x d^{2} y\left(\lambda \delta^{2}(x-y)-\frac{\epsilon}{\Lambda^{2}} \partial_{1}^{2} \delta^{2}(x-y)\right) \cos \sqrt{4 \pi}(\phi(x)+\phi(y)) \\
= & S_{\text {int }}^{\prime} .
\end{aligned}
$$


As usual, imposing the invariance of the action under RG, we get the flow equations for the couplings $\lambda$ and $\epsilon$ :

$$
\begin{aligned}
& \frac{d \lambda}{d t}=(2-4 K) \lambda-2 K \epsilon, \\
& \frac{d \epsilon}{d t}=-4 K \epsilon,
\end{aligned}
$$

where $\ln s=t$, with the initial conditions $\lambda(0)=\lambda_{0}$ and $\epsilon(0)=\epsilon_{0}$. The solution of this system is elementary, yielding:

$$
\begin{aligned}
& \lambda(t)=\left(\lambda_{0}-K \epsilon_{0}\right) \exp [(2-4 K) t]+K \epsilon_{0} \exp [-4 K t], \\
& \epsilon(t)=\epsilon_{0} \exp [-4 K t] .
\end{aligned}
$$

From the last equation one clearly sees that the non-local piece of the interaction is irrelevant, as expected. Concerning the local interaction one sees that it is relevant for $K<1 / 2$, i.e., non-locality does not modify this well-known condition already found for the local SG model. Therefore, for $K<1 / 2, \lambda$ will grow with increasing $t$ and there will be a gap in the CD spectrum which can be estimated by determining the value $\tilde{t}$ for which $\lambda=1$. From now on we shall restrict our analysis to the case $K<1 / 2$. The gap is then given by $\Delta E=\Lambda \mu e^{-\tilde{t}}$, where $\mu=c /(\pi \sqrt{2}) \approx 0.198$. It is also convenient to define the dimensionless gap $m=\Delta E / \Lambda$. From Eq. (3.10) one can thus derive an equation for $m$ which gives the behavior of the energy gap as function of the forward scattering potentials $(K)$ and the non-local contribution of the umklapp scattering $\left(\epsilon_{0}\right)$. Before analyzing this non-trivial equation it seems reasonable to check if it predicts sensible results for the local case. To this end we set $\epsilon_{0}=0$ and $\lambda=1$ in (3.10), obtaining:

$$
m_{0}=\mu \lambda_{0}^{\frac{1}{2-4 K}} \text {. }
$$

This result can be compared with the exact solution obtained by Zamolodchikov [22] and with the approximated result given by the SCHA method [13]. The corresponding expressions for the gap are respectively given by:

$$
m_{Z}=\frac{2^{\frac{-2 K}{1-2 K}}}{\sqrt{\pi}} \mu^{\frac{1}{1-2 K}} \frac{\Gamma\left(\frac{K}{1-2 K}\right)}{\Gamma\left(\frac{1}{2-4 K}\right)}\left(\pi \frac{\Gamma(1-2 K)}{\Gamma(2 K)}\right)^{\frac{1}{2-4 K}} \lambda_{0}^{\frac{1}{2-4 K}}
$$

and

$$
m_{\text {scha }}=\frac{2}{\sqrt{K}}(4 \pi K)^{\frac{1}{2-4 K}} \mu^{\frac{1}{1-2 K}} \lambda_{0}^{\frac{1}{2-4 K}} .
$$

In order to compare $m_{0}$ and $m_{\text {scha }}$ with $m_{Z}$ in an efficient and easy to visualize way, we have computed the relative error $\Delta m / m_{Z}$ as function of $K$ for both approximations. The results are depicted in Fig. 1 where one sees that our RG computation gives values of the gap closer to the exact values for a wide range of the stiffness constant $K$. Interestingly, the SCHA result works well when one approaches the end points of the interval.

Going back to the case $\epsilon_{0} \neq 0$, by combining Eqs. (3.8) and (3.9) one readily gets a phase diagram in the $\epsilon-\lambda$ plane (see Fig. 2). There is a critical line given by $\lambda=K \epsilon$. If 


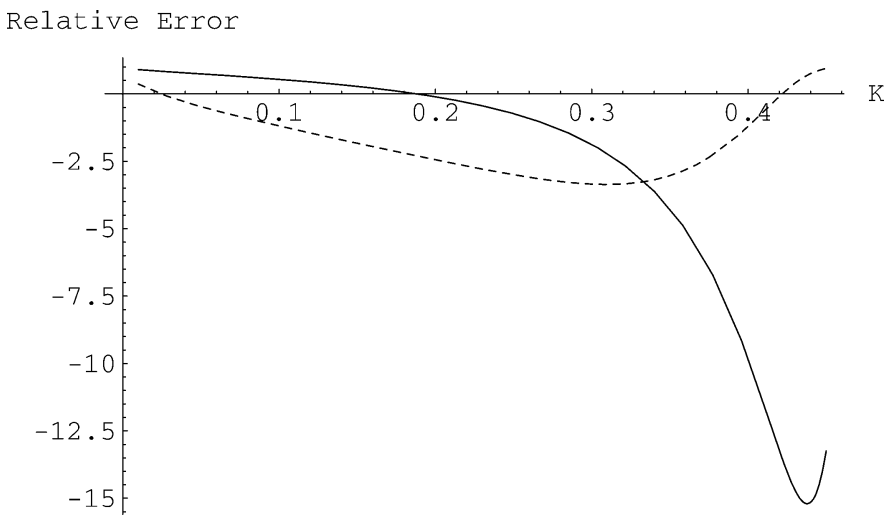

Fig. 1. Relative error $\Delta m / m$ as function of the stiffness constant, for the local case. The dashed line corresponds to the SCHA whereas the solid line shows the RG result.

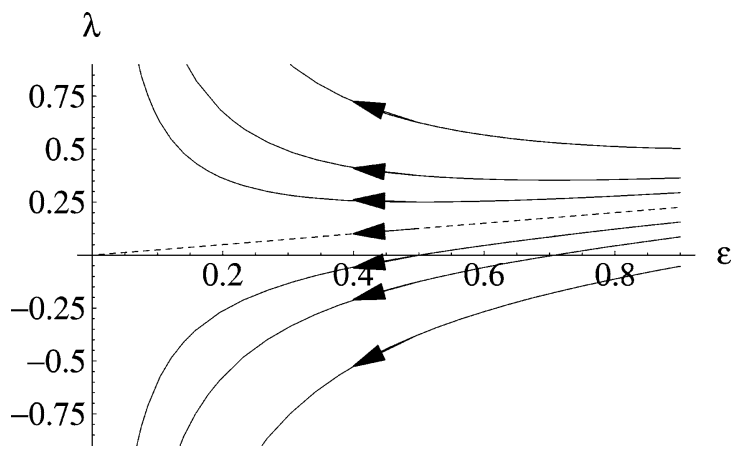

Fig. 2. Phase diagram in the $\epsilon-\lambda$ plane, for $K=0.25$. The dashed line is the critical line $\lambda=K \epsilon$.

the initial parameters are tuned to lie on this line, the system will flow to the TomonagaLuttinger fixed point, at the origin. In this case, of course, the system remains gapless. On the other hand, for initial conditions outside the critical line, the system flows to strong coupling, giving rise to a gap $m$, as mentioned above. For simplicity let us consider the case $\lambda>\epsilon$ and define the variable $x=m / m_{0}$. The gap equation can then be written as

$$
x^{2}-\frac{v}{\epsilon_{0}} x^{2-4 K}+\frac{v}{\epsilon_{0}}\left(1-\frac{K \epsilon_{0}}{\lambda_{0}}\right)=0,
$$

where $v=\lambda_{0}^{\frac{-2 K}{1-2 K}} / K$. This equation is one of our main results. For fixed values of $K$ and $\lambda_{0}$ it gives the behaviour of the energy gap as function of the non-local contribution to umklapp scattering, associated to non-contact interactions. The form of this formula suggests that it could be easier to handle the inverted equation:

$$
\epsilon_{0}(x)=\epsilon_{\text {crit }} \nu \frac{1-x^{2-4 K}}{v-\epsilon_{\text {crit }} x^{2}},
$$




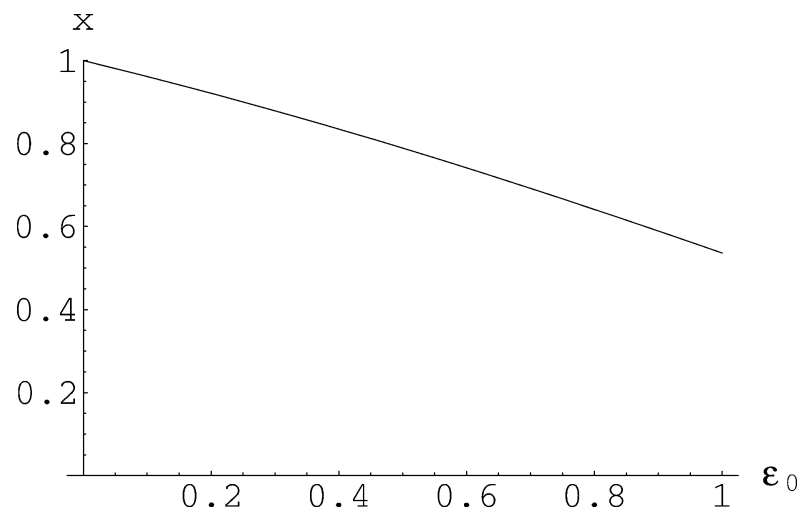

Fig. 3. Numerical solution of the gap equation for $\lambda_{0}=0.5$ and $K=0.25$. The gap $m$ decreases for increasing $\epsilon_{0}$.

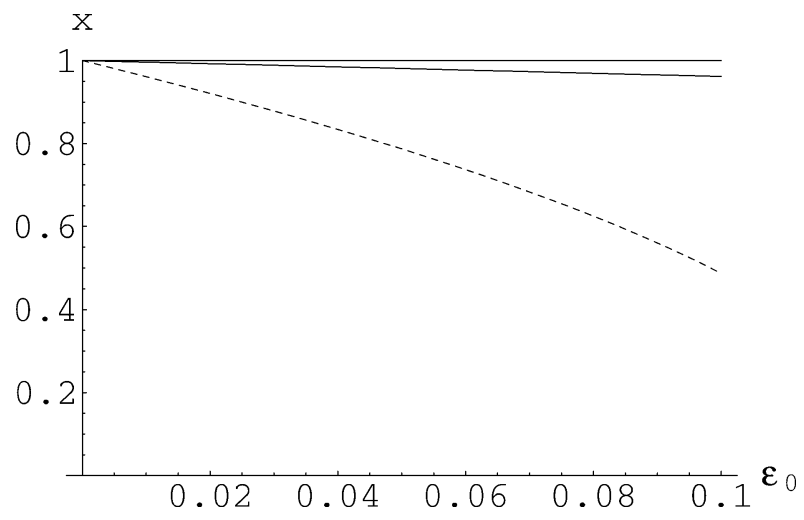

Fig. 4. Numerical solutions of the gap equations for $\lambda_{0}=0.5, K=0.25$, and $0 \leqslant \epsilon_{0} \leqslant 0.1$. The dashed line corresponds to the SCHA whereas the solid line shows the RG result. The unit function is included to allow comparison with the local case.

where $\epsilon_{\text {crit }}=\lambda_{0} / K$. In Fig. 3 we show the numerical solution of this last equation for $\lambda_{0}=0.5$ and $K=0.25$. We see that $x$ decreases for increasing $\epsilon_{0}$, in qualitative agreement with the SCHA prediction [13]. A quantitative comparison between both approximations is given in Fig. 4, for the same fixed values of $\lambda_{0}$ and $K$. Since the SCHA result obtained in [13] is valid for small values of the coefficient associated to non-locality, we have plotted the solutions in the interval $0 \leqslant \epsilon_{0} \leqslant 0.1$. We find that the gap decay predicted by $\mathrm{RG}$, for increasing non-locality, is much slower than the one obtained through the Gaussian approximation.

As a final comment, we note that the RG treatment for non-local interactions depicted in this work can be extended to a more general coupling function including an arbitrary 
number of even powers of $p_{1}$. In coordinate space such an interaction can be written as

$$
g_{3}(x)=\sum_{i=0}^{n}(-1)^{i} \frac{\lambda_{i}}{\Lambda^{2 i}} \partial^{2 i} \delta(x),
$$

where $\lambda_{1}=\epsilon_{0}$. The corresponding set of RG equations for the $\lambda_{i}^{\prime} s$ can be obtained from the expansion of the cosine integral function which appears when one integrates the fast modes after the mode separation (see Appendix A). The result is

$$
\begin{aligned}
& \frac{d \lambda_{0}}{d t}=(2-4 K) \lambda_{0}-2 K \sum_{i=1}^{n} \lambda_{i} \\
& \vdots \\
& \frac{d \lambda_{j}}{d t}=(2-2 j-4 K) \lambda_{j}-2 K \sum_{i=j+1}^{n} \lambda_{i} \\
& \vdots \\
& \frac{d \lambda_{n}}{d t}=(2-2 n-4 K) \lambda_{n} .
\end{aligned}
$$

The general solution of this system can be expressed as a combination of exponentials and the computation of the gap cannot be done, in principle, in an analytical way. It is then illustrative to consider a particular case in which this calculation is simplified. Indeed, for small $t$, it can be proved by induction that the solutions of this system of equations are of the form:

$$
\lambda_{j}(t)=\lambda_{j}^{0} \exp \left[t\left(2-2 j-4 K-\frac{2 K}{\lambda_{j}^{0}} \sum_{i=j+1}^{n} \lambda_{i}^{0}\right)\right] .
$$

In this limit one obtains the following expression for the gap:

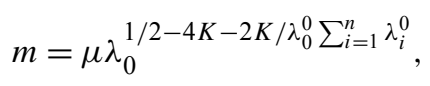

which is consistent with the conditions $\lambda_{0} \sim 1^{-}$and $K \ll 1 / 2$.

\section{Conclusions}

In this paper we have considered a non-local extension of the sine-Gordon model. This theory is obtained when one bosonizes a non-local and non-covariant version of the Thirring model used to describe certain 1d many-body systems. Since the integrability of this non-local sine-Gordon model has not been proved, one needs to implement some approximation to study its physical content. We have performed a RG calculation up to first order in the coupling function $g_{3}$, which in a condensed matter context is associated to the so-called umklapp scattering. We obtained an expression for the energy gap as function of the non-local piece of the interaction $\epsilon_{0}$. For purely local interactions (the exactly solvable $\mathrm{SG}$ ) our result seems to be a sensible approximation, improving the 
SCHA predictions for a wide range of forward interactions. In the non-local case, in which no exact answer is known, we predict decreasing values for the gap for increasing values of $\epsilon_{0}$, in qualitative agreement with a previous SCHA computation. We were able to give a precise comparison between both approximations in the interval $0 \leqslant \epsilon_{0} \leqslant 0.1$, showing that the gap decrease, for increasing non-locality, is much weaker according to the RG computation. Since, as is well known, the SCHA method is not a controlled approximation, the present results contribute to a better understanding of the physics of non-local field theories. We think that our results are also of interest in the context of 1D many-body systems (Luttinger liquids) in which most of the previous investigations involving umklapp scattering do not consider non-local effects associated to long range interactions [23].

\section{Acknowledgements}

This work was partially supported by Universidad Nacional de La Plata and Consejo Nacional de Investigaciones Científicas y Técnicas, CONICET (Argentina).

\section{Appendix A}

In order to illustrate the computation leading to the flow equations we first define the free bosonic propagator:

$$
\langle\phi(k) \phi(q)\rangle_{0}=\frac{1}{Z_{0}} \int D \phi \phi(k) \phi(q) e^{-S_{0}}=\left.\frac{1}{Z_{0}} \frac{\delta^{2} Z(j)}{\delta j(k) \delta j(q)}\right|_{j=0}
$$

with

$$
Z(j)=\int D \phi \exp \left[-\int \frac{d^{2} p}{2(2 \pi)^{2}}(\Phi(p) F(p) \phi(-p)-J(p) \phi(p))\right] .
$$

The result is

$$
\langle\phi(p) \phi(q)\rangle_{0}=\frac{\delta^{2}(p+q) 4 \pi^{2} K v}{p_{0}^{2}+v^{2} p_{1}^{2}} .
$$

The next step is the analysis of $S_{\text {int }}$, as given by the second term of Eq. (3.1). For simplicity, in this appendix we disregard the overall constant $(\Lambda c)^{2} /\left(2 \pi^{2}\right)$. Going to momentum space and performing the separation in slow and fast modes $\phi_{<}$and $\phi_{>}$, according to:

$$
\begin{aligned}
& \phi(x)=\frac{1}{(2 \pi)^{2}} \int d^{2} p \phi(p) e^{i p \cdot x}, \\
& \phi(p)=\phi_{<}(p), \quad\left|p_{1}\right|<\frac{\Lambda}{s},
\end{aligned}
$$




$$
\phi(p)=\phi_{>}(p), \quad \frac{\Lambda}{s} \leqslant\left|p_{1}\right| \leqslant \Lambda,
$$

we obtain

$$
\begin{aligned}
S_{\mathrm{int}}=\int d^{2} x d y_{1} g_{3}\left(x_{1}-y_{1}\right) \\
\times\left(\cos \left[\frac{\sqrt{4 \pi}}{(2 \pi)^{2}} \int d^{2} p \phi_{<}(p) f\left(p, x, y_{1}\right)\right]\right. \\
\quad \times \cos \left[\frac{\sqrt{4 \pi}}{(2 \pi)^{2}} \int d^{2} p \phi_{>}(p) f\left(p, x, y_{1}\right)\right] \\
\quad-\sin \left[\frac{\sqrt{4 \pi}}{(2 \pi)^{2}} \int d^{2} p \phi_{<}(p) f\left(p, x, y_{1}\right)\right] \\
\left.\quad \times \sin \left[\frac{\sqrt{4 \pi}}{(2 \pi)^{2}} \int d^{2} p \phi_{>}(p) f\left(p, x, y_{1}\right)\right]\right),
\end{aligned}
$$

where

$$
f\left(p, x, y_{1}\right)=e^{i p \cdot x}+e^{i\left(p_{0} x_{0}+p_{1} x_{1}\right)} .
$$

Now we expand the functional integral up to first order in $g_{3}$ and integrate over the fast modes. One finds the following results:

$$
\left\langle\sin \left[\frac{\sqrt{4 \pi}}{(2 \pi)^{2}} \int d^{2} p \phi_{>}(p) f\left(p, x, y_{1}\right)\right]\right\rangle_{0}=0
$$

and

$$
\begin{aligned}
\left\langle\cos \left[\frac{\sqrt{4 \pi}}{(2 \pi)^{2}} \int d^{2} p \phi_{>}(p) f\left(p, x, y_{1}\right)\right]\right\rangle_{0} \\
\quad=\exp \left(-K \int d p_{1}\left(\theta\left(p_{1}\right)-\theta\left(-p_{1}\right)\right) \frac{1+\cos \left(p_{1}\left(x_{1}-y_{1}\right)\right)}{p_{1}}\right) \\
\quad=\exp \left(-K\left[2 \ln s+2 C i\left(\Lambda\left(x_{1}-y_{1}\right)\right)-2 C i\left(\frac{\Lambda}{s}\left(x_{1}-y_{1}\right)\right)\right]\right),
\end{aligned}
$$

where $C i(x)$ is the cosine integral function and the free vacuum expectation values are, of course, taken with respect to fast modes. Rescaling momenta, coordinates and fields such that the free piece of the action $S_{0}$ remains invariant:

$$
p^{\prime}=s p, \quad x^{\prime}=s^{-1} x, \quad \phi^{\prime}\left(p^{\prime}\right)=s^{-2} \phi_{<}\left(p^{\prime} / s\right),
$$

and using the fact that $\ln s=t, s \simeq 1+t, S_{\text {int }}$ can be written as

$$
\begin{aligned}
S_{\text {int }}= & \int d^{2} x^{\prime} d y_{1}^{\prime} g_{3}\left(s\left(x_{1}^{\prime}-y_{1}^{\prime}\right)\right) \cos \left[\frac{\sqrt{4 \pi}}{(2 \pi)^{2}} \int d^{2} p \phi_{<}(p) f\left(p, x, y_{1}\right)\right] \\
& \times\left(1+\left[(3-4 K)+K\left(\Lambda\left(x_{1}^{\prime}-y_{1}^{\prime}\right)\right)^{2}-\frac{K}{12}\left(\Lambda\left(x_{1}^{\prime}-y_{1}^{\prime}\right)\right)^{4}\right] t\right),
\end{aligned}
$$


where we have used the power expansion of the function $C i(x)$. Finally, using the explicit expression for $g_{3}$ in terms of $\lambda_{0}$ and $\epsilon_{0}$ we obtain

$$
\begin{aligned}
S_{\text {int }}=\int d^{2} x^{\prime} d^{2} y^{\prime}[ & \left(\lambda_{0}+\lambda_{0}[2-4 K] t-2 K \epsilon_{0} t\right) \delta^{2}\left(x_{1}^{\prime}-y_{1}^{\prime}\right) \\
& \left.-\frac{\epsilon_{0}-4 K \epsilon_{0} t}{\Lambda^{2}} \partial_{1}^{2} \delta^{2}\left(x_{1}^{\prime}-y_{1}^{\prime}\right)\right] \cos \sqrt{4 \pi}\left[\phi\left(x^{\prime}\right)+\phi\left(y^{\prime}\right)\right] \\
= & \int d^{2} x^{\prime} d^{2} y^{\prime}\left[\lambda^{\prime} \delta^{2}\left(x_{1}^{\prime}-y_{1}^{\prime}\right)-\frac{\epsilon^{\prime}}{\Lambda^{2}} \partial_{1}^{2} \delta^{2}\left(x_{1}^{\prime}-y_{1}^{\prime}\right)\right] \cos \sqrt{4 \pi}\left[\phi\left(x^{\prime}\right)+\phi\left(y^{\prime}\right)\right] \\
= & S_{\text {int }}^{\prime},
\end{aligned}
$$

which leads to the flow equations for $\lambda$ and $\epsilon$.

\section{References}

[1] R.P. Feynman, J.A. Wheeler, Rev. Mod. Phys. 17 (1945) 157.

[2] D.A. Eliezer, R.P. Woodard, Nucl. Phys. B 325 (1989) 389.

[3] E.C. Marino, Phys. Lett. B 263 (1991) 63;

E.C. Marino, Nucl. Phys. B 408 (1993) 551.

[4] D.G. Barci, C.D. Fosco, L. Oxman, Phys. Lett. B 375 (1996) 267;

D.G. Barci, L. Oxman, Mod. Phys. Lett. A 12 (1997) 493.

[5] I.V. Krive, et al., Phys. Rev. B 52 (1995) 10865.

[6] C. Naón, M.C. von Reichenbach, M.L. Trobo, Nucl. Phys. B 435 [FS] (1995) 567;

C. Naón, M.C. von Reichenbach, M.L. Trobo, Nucl. Phys. B 485 [FS] (1997) 665;

M. Manías, C. Naón, M.L. Trobo, Nucl. Phys. B 525 [FS] (1998) 721;

V.I. Fernández, C.M. Naón, Teor. Mat. Fiz. 118 (1999) 385 (in Russian), hep-th/9810048.

[7] S. Iijima, Nature 354 (1991) 56;

T.W. Ebbesen, Phys. Today 49 (6) (1996) 26.

[8] H. Otani, T. Ogawa, Phys. Rev. B 54 (1996) 4540.

[9] D. Poilblanc, et al., Phys. Rev. B 56 (1997) R1645.

[10] G. Fano, et al., Phys. Rev. B 60 (1999) 15654;

S. Capponi, et al., Phys. Rev. B 61 (2000) 13410.

[11] R. Egger, A.O. Gogolin, Phys. Rev. Lett. 79 (1997) 5082.

[12] B. Dumoulin, et al., Phys. Rev. Lett. 76 (1996) 1360.

[13] V.I. Fernández, A. Iucci, C.M. Naón, Nucl. Phys. B 636 [FS] (2002) 514, hep-th/0112057.

[14] W. Thirring, Ann. Phys. 3 (1958) 91;

B. Klaiber, in: A. Barut, W. Brittin (Eds.), in: Lectures in Theoretical Physics, Boulder, Vol. 10A, Gordon and Breach, New York, 1967, p. 141;

V.E. Korepin, Teor. Mat. Fiz. 41 (1979) 169 (in Russian).

[15] J. Solyom, Adv. Phys. 28 (1979) 209.

[16] A. Luther, V.J. Emery, Phys. Rev. Lett. 33 (1974) 589;

R. Heidenreich, B. Schroer, R. Seiler, D.A. Uhlenbrock, Phys. Lett. A 54 (1975) 119.

[17] V.J. Emery, A. Luther, I. Peschel, Phys. Rev. B 13 (1976) 1272.

[18] Y. Saito, Z. Phys. B 32 (1978) 75;

M.P.A. Fisher, W. Zwerger, Phys. Rev. B 32 (1985) 6190;

M. Stevenson, Phys. Rev. D 32 (1985) 1389;

R. Ingermanson, Nucl. Phys. B 266 (1986) 620;

A. Iucci, C.M. Naón, J. Phys. B 35 (2002) 8005, hep-th/0209019.

[19] R. Shankar, Rev. Mod. Phys. 66 (1994) 129;

H.J. Schulz, G. Cuniberti, P. Pieri, cond-mat/9807366;

S. Rao, D. Sen, cond-mat/0005492. 
[20] S. Tomonaga, Prog. Theor. Phys. 5 (1950) 544;

J. Luttinger, J. Math. Phys. 4 (1963) 1154;

E. Lieb, D. Mattis, J. Math. Phys. 6 (1965) 304.

[21] A. Iucci, K. Li, C.M. Naón, Nucl. Phys. B 601 [FS] (2001) 607, hep-th/0012269.

[22] Al.B. Zamolodchikov, Int. J. Mod. Phys. A 10 (1995) 1125.

[23] See, for instance, U. Zülicke, Phys. Rev. Lett. 83 (1999) 5330;

A. Rosch, cond-mat/0104181, Adv. Solid State Phys. 41, in press. 